\title{
Comparing durum wheat cultivars by genotype $\times$ yield $\times$ trait and genotype $\times$ trait biplot method
}

\author{
Enver Kendal $^{1 *}$ \\ ${ }^{1}$ Mardin Artuklu University, Kiziltepe Vocational Training High School, Postal code: 47060, Mardin, Turkey. \\ "Corresponding author(enver21_1@hotmail.com).
}

Received: 12 March 2019; Accepted: 6 June 2019; doi:10.4067/S0718-58392019000400512

\begin{abstract}
The specification of the most convenient cultivars based on multiple trait indices is a new approach in durum wheat (Triticum durum Desf.) adaptation and stability studies. This approach helps to define the best cultivar based on multiple traits and multiple locations because cultivars are affected by unpredictable climatic conditions. Some traits (ears per square meter, spike length, number of grains per spike, spike yield, and leaf chlorophyll content among others) can be produced for primary breeding purposes because they are influenced by environmental factors and indirectly affect grain yield and quality. Therefore, in the present study, the new genotype $\times$ yield $\times$ trait (GYT) biplot approach was used to identify the best cultivar among 10 durum wheat cultivars based on multiple environments (8) and multiple traits (18). Cultivar ranking was examined by a superiority index that combined yield and other target traits with the GYT biplot. The general adaptability of each cultivar in terms of all the traits indicated differences based on environment means, and significant differences were found between varieties for the GYT biplot. In the GYT biplot, yield-trait combinations clearly indicated the most stable cultivars, whereas in the genotype $\times$ trait (GT) biplot, the best cultivars were not defined for all traits. 'Sariçanak' was ranked as the best combination of physio-morphological traits with grain yield, 'Zühre' was the best for more quality traits, and 'Güneyyildizi' was the best for both physio-morphological and quality traits in the GYT biplot. The GYT biplot combines traits with yield and can help the visual identification of the best cultivars; it is better than the GT biplot method.
\end{abstract}

Key words: Durum, cultivar, multiple, environment, trait, genotype $\times$ yield $\times$ trait.

\section{INTRODUCTION}

Durum wheat (Triticum durum Desf.) is a very significant crop for different industries (bulgur, macaroni, and cake) and nearly 40 to 50 million tons are produced every year worldwide. The 28-nation European Union is the leading producing community with 9.5 million tons, followed by Canada with 7.8 million tons. Turkey is also an important durum wheat producer with close to 4 million tons annually. Approximately half of the durum wheat production in Turkey takes place in the Southeastern Anatolia Region, known as the central origin of durum wheat. Since the agroecological environmental conditions of this region are very suitable for wheat production, the highest quality durum wheat has been produced in this region; this situation has been established by many researchers (Kendal and Dogan, 2015; Kilic et al., 2017; Tekdal and Kendal, 2018).

The new registered varieties need to be well adapted to both high-yield and a broad range of environments. Many different methods have been developed to characterize the behavior of varieties under different environmental conditions. Yield trials in different environments provide information about the Genotype $\times$ Environment (GE) interaction when 
analyzed by traditional methods. However, it is very difficult to achieve this in areas where the cultivation of durum wheat is intense under the influence of the Mediterranean climate because the GE interaction is quite high. During the growing period, many ecological and agronomic problems are encountered in the cultivation activities that limit the success of researchers, who must therefore struggle to develop different models to overcome these problems (Mohammadi et al., 2015; Giunta et al., 2018; Karaman, 2019).

There are two major problems in assessing cultivars. The first is the negative GE interaction and the second deals with basic traits (Yan and Frégeau-Reid, 2018). The first issue for researchers is to identify the cultivars that are not affected by the GE interaction and that are stable. For this purpose, the GE interaction and the additive main effect and multiplicative interaction (AMMI) have been developed to characterize the behavior of varieties under different environmental conditions. Therefore, many researchers have been working with durum wheat in different years and environments (Kilic, 2014; Baxevanos et al., 2017; Magallanes-López et al., 2017; Mohammadi et al., 2018; Sadeghzadeh et al., 2018). The second issue is the registration of new varieties that could produce the best outputs (high yield and quality) under different environmental conditions (drought and heat stress, resistance to diseases, frost risk, and irregular rainfall). It is very difficult to improve the best varieties in terms of all studied traits in different environments (Pinheiro et al., 2013; Sissons et al., 2014; Mohammadi et al., 2015). In breeding studies, when trying to develop a cultivar trait, it is possible to disrupt another trait. For this reason, the genotype $\times$ yield $\times$ trait (GYT) biplot evaluates all the traits together and provides more accurate results. In this regard, durum wheat breeders should know whether any trait is negatively or positively correlated with grain yield. Heading time, ears per square meter, spike length, number of grains per spike, spike yield, and leaf chlorophyll content are very important traits to identify if a durum wheat variety is resistant to drought, heat stress, cold damage, what is the plant height for lodging, as well as protein content, 1000-grain yield, and hectoliter weight for quality in the Southeastern Anatolia Region of Turkey.

Researchers have been using the genotype $\times$ trait (GT) biplot technique in breeding for a long time. However, this method cannot provide good outputs for breeders to know which cultivar to recommend, select, or eliminate. The GYT biplot technique was therefore designed to mitigate the deficiencies encountered in the GT biplot technique and facilitate a more efficient selection for plant breeders. This technique is used to identify cultivars according to their general advantages over yield-trait combinations and show trait profiles. The aim of this study was to assess cultivar evaluation and trait profiles of durum wheat using the GYT and GT biplot techniques and identify the target traits by combining yield with GYT and comparing with the GT biplot technique to observe the advantages of the GYT biplot based on multiple traits and multiple environments.

\section{MATERIALS AND METHODS}

Ten spring durum cultivars were evaluated at eight locations across 2 yr (2011-2012 and 2012-2013 growing seasons). Cultivar information is shown in Table 1 and locations in Table 2.

The trials were conducted using a randomized block design with four replicates and a sowing density of $500 \mathrm{seeds}^{-2}$. Plot size was $7.2 \mathrm{~m}^{2}(6 \mathrm{~m} \times 1.2 \mathrm{~m})$ with six rows and $20 \mathrm{~cm}$ row spacing. Trials were sown in autumn. Fertilization was $60 \mathrm{~kg} \mathrm{~N} \mathrm{ha}^{-1}$ and $60 \mathrm{~kg} \mathrm{Pha}^{-1}$ at sowing and $60 \mathrm{~kg} \mathrm{~N}$ ha-1 $^{-1}$ at tillering for all plots. A $6 \mathrm{~m}^{2}$ area in each plot was harvested with a Hege 140 harvester (Hans Ulrich Hege, Waldenburg, Germany).

Table 1. Durum wheat code, name, origin, and registration year.

\begin{tabular}{lllc}
\hline & \multicolumn{1}{c}{ Name } & \multicolumn{1}{c}{ Origin } & Year \\
\hline C1 & Artuklu & GAP International Agricultural Research and Training Center & 2008 \\
C2 & Aydin & GAP International Agricultural Research and Training Center & 1993 \\
C3 & Eyyubi & GAP International Agricultural Research and Training Center & 2008 \\
C4 & Güneyyildizi & GAP International Agricultural Research and Training Center & 2010 \\
C5 & Harran & GAP International Agricultural Research and Training Center & 1995 \\
C6 & Sariçanak & GAP International Agricultural Research and Training Center & 1998 \\
C7 & Svevo & Agricultural Liability Company & 2001 \\
C8 & Şahinbey & GAP International Agricultural Research and Training Center & 2008 \\
C9 & Zenit & Agricultural Liability Company & 2001 \\
C10 & Zühre & GAP International Agricultural Research and Training Center & 2010 \\
\hline
\end{tabular}


Table 2. Years, sites, codes, and geographic coordinates.

\begin{tabular}{llcccc}
\hline Years & Sities & Altitude & Latitude & Longitude & Mean water supply \\
\hline \multirow{2}{*}{$2011-2012$} & m a.s.l. & & & $\mathrm{mm}$ \\
& Diyarbakir & 612 & $37^{\circ} 55^{\prime} \mathrm{N}$ & $40^{\circ} 14^{\prime} \mathrm{E}$ & 483.5 rainfall \\
$2012-2013$ & Diyarbakir & 612 & $37^{\circ} 55^{\prime} \mathrm{N}$ & $40^{\circ} 14^{\prime} \mathrm{E}$ & $483.5+200$ irrigated \\
& Hazro & 995 & $38^{\circ} 24^{\prime} \mathrm{N}$ & $40^{\circ} 24^{\prime} \mathrm{E}$ & 891.9 rainfall \\
& Kiziltepe & 484 & $37^{\circ} 19^{\prime} \mathrm{N}$ & $40^{\circ} 58^{\prime} \mathrm{E}$ & $231.3+400$ irrigated \\
\hline
\end{tabular}

Moreover, traits such as yield, heading time (HD), stalks per square meter (SS), ears per square meter (ES), maturation time (MT), plant height (PH), spike length (SL), number of spikelets per spike (NSS), number of grains per spike (NGS), spike yield (SY), leaf chlorophyll content (SPAD), vitreous kernels (VIT), hectoliter weight (HW), 1000-grain weight (TGW), protein content (PC), semolina color (SC), mini sedimentation (SDS), and wet gluten (WG), were studied in 10 durum wheat cultivars across $2 \mathrm{yr}$.

\section{Statistical analysis (genotype $\times$ yield $\times$ trait and genotype $\times$ trait)}

The data of 10 durum wheat cultivars in multi-location and multi-year trials were analyzed to determine whether the effect of the Genotype $\times$ Environment $(\mathrm{GE})$ interaction was significant; means were separated using the least significant differences (LSD) test with significance set at $\mathrm{P}<0.05$ and $\mathrm{P}<0.01$. Data were also graphically analyzed by the genotype $\times$ trait (GT) biplot method as recommended by Yan and Thinker (2005), and the genotype $\times$ yield $\times$ trait (GYT) biplot method as recommended by Yan and Frégeau-Reid (2018). A superiority index (SI) combining all Yield-Trait interactions was calculated based on the standardized GYT (Yan and Frégeau-Reid, 2018).

Data standardization. The GT or GYT tables were standardized so that the mean for each trait or yield-trait combination was 0 and the variance a unit (see Table 5). Standardization was performed as:

$$
P i j=\frac{T i j-T j}{S j}
$$

where $P i j$ is the standardized value of genotype $\mathrm{i}$ for trait or yield-trait combination $\mathrm{j}$ in the standardized table, Tij is the original value of genotype $i$ for trait or yield-trait combination $\mathrm{j}$ in the GT or GYT tables (Tables 3 and 6 ), $T j$ is the mean across genotypes for trait or yield-trait combination $\mathrm{j}$, and $S \mathrm{j}$ is the standard deviation for trait or yield-trait combination $\mathrm{j}$.

The biplot method was constructed for all scored genotype traits with the GenStat 14th Edition release software program (GenstatlVSN International). Data were graphically analyzed to interpret GT and GYT with the GGE biplot software.

\section{RESULTS}

The combined ANOVA revealed significant differences among environments for all traits $(\mathrm{P}<0.01, \mathrm{P}<0.05)$ and highly significant differences $(\mathrm{P}<0.01, \mathrm{P}<0.05)$ were recorded among the genotypes for all investigated traits (Table 3 ).

The mean yield (YLD) of genotypes across environments varied between 5582 and $6506 \mathrm{~kg} \mathrm{ha}^{-1}$, HD between 124 and $126 \mathrm{~d}$, SS between 572 and 643 stalks m$^{-2}$, ES between 443 and 530 ears m$^{-2}$, MT between 163 and $165 \mathrm{~d}$, PH between 91 and $106 \mathrm{~cm}$, SL between 6.0 and $7.5 \mathrm{~cm}$, NSS between 19.0 and 20.7, NGS between 45 and 55, SY between 2.0 and $2.3 \mathrm{~g}$, leaf chlorophyll content between 47 and $51 \mathrm{SPAD}$, VIT between $87 \%$ and $99 \%$, HW between 82 and $85 \mathrm{~kg} \mathrm{hL}^{-1}$, TGW between 41 and $50 \mathrm{~g}$, PC between $13.3 \%$ and 14.6\%, SC between 19 and 23, SDS between 4.7 and $7.8 \mathrm{~mL}$, and WG between 25 and 29 based on means across environments.

\section{The genotype $\times$ trait biplot}

Genotype $\times$ trait biplot analysis (GT biplot) is highlighted among the multivariate methodologies because it assesses genotypes based on multiple traits and identifies those that are superior to the desired variables; these can be used as parents in breeding programs or even as possible commercial cultivars. A quick and practical visualization of the genetic correlation between traits is also provided by this analysis. Less important (redundant) traits can be detected and identified as the most suitable to indirectly select a favorable trait (Yan and Tinker, 2006). Trait values across $2 \mathrm{yr}$ in four locations 
Table 3. Mean data for 2 years in four environments of 10 durum wheat cultivars.

\begin{tabular}{|c|c|c|c|c|c|c|c|c|c|c|c|c|}
\hline \multirow[b]{2}{*}{ Traits } & \multicolumn{10}{|c|}{ Cultivars } & \multirow[b]{2}{*}{ Mean } & \multirow[b]{2}{*}{ LSD } \\
\hline & $\mathrm{C} 1$ & $\mathrm{C} 2$ & $\mathrm{C} 3$ & $\mathrm{C} 4$ & C5 & C6 & C7 & C8 & C9 & $\mathrm{C} 10$ & & \\
\hline $\mathrm{HD}, \mathrm{d}$ & 125 & 126 & 124 & 125 & 125 & 124 & 121 & 124 & 124 & 124 & 124 & $2.49 * *$ \\
\hline $\mathrm{SS}, \mathrm{m}^{2}$ & 572 & 586 & 572 & 613 & 587 & 585 & 643 & 568 & 611 & 597 & 593 & $20.63 * *$ \\
\hline $\mathrm{ES}, \mathrm{m}^{2}$ & 459 & 487 & 488 & 488 & 490 & 477 & 530 & 443 & 492 & 504 & 486 & $17.60 * *$ \\
\hline MT, d & 165 & 166 & 164 & 165 & 166 & 165 & 163 & 166 & 166 & 165 & 165 & $0.56^{* *}$ \\
\hline $\mathrm{PH}, \mathrm{cm}$ & 103 & 106 & 98 & 97 & 91 & 89 & 94 & 93 & 88 & 94 & 95 & $1.88 * *$ \\
\hline $\mathrm{SL}, \mathrm{cm}$ & 7.1 & 6.3 & 6.8 & 6.7 & 6.8 & 6.6 & 6.0 & 6.9 & 7.5 & 6.8 & 6.7 & $0.17 * *$ \\
\hline NSS & 19.9 & 20.5 & 19.9 & 19.0 & 20.5 & 20.7 & 17.7 & 20.3 & 20.7 & 19.8 & 20 & $0.49^{*}$ \\
\hline NGS & 51 & 51 & 51 & 50 & 50 & 55 & 45 & 46 & 45 & 49 & 49 & $1.66 * *$ \\
\hline SY, g & 2.3 & 2.1 & 2.3 & 2.2 & 2.2 & 2.3 & 2.0 & 2.2 & 2.0 & 2.0 & 2.2 & $0.03 * *$ \\
\hline SPAD & 48 & 48 & 51 & 45 & 47 & 48 & 47 & 47 & 47 & 47 & 48 & $0.56 * *$ \\
\hline VIT, \% & 94 & 92 & 92 & 96 & 87 & 88 & 99 & 93 & 97 & 99 & 94 & $0.14 * *$ \\
\hline $\mathrm{HW}, \mathrm{kg} \mathrm{hL}^{-1}$ & 84 & 85 & 85 & 84 & 82 & 85 & 84 & 83 & 83 & 84 & 84 & $0.82 * *$ \\
\hline TGW, g & 45 & 42 & 46 & 44 & 47 & 43 & 43 & 50 & 44 & 41 & 45 & $1.14 * *$ \\
\hline $\mathrm{PC}, \%$ & 13.7 & 14.2 & 13.5 & 14.2 & 14.0 & 13.3 & 14.6 & 13.6 & 14.3 & 14.1 & 14.0 & $0.21 * *$ \\
\hline SC & 20 & 21 & 20 & 22 & 22 & 21 & 23 & 19 & 24 & 22 & 21 & $0.48 * *$ \\
\hline SDS, mL & 5.6 & 5.2 & 7.2 & 7.0 & 6.2 & 4.2 & 7.9 & 4.5 & 7.8 & 7.8 & 6.4 & $0.38 * *$ \\
\hline WG & 27 & 28 & 27 & 28 & 27 & 25 & 29 & 26 & 28 & 28 & 27 & $0.73 * *$ \\
\hline YLD, $\mathrm{kg} \mathrm{ha}^{-1}$ & 5906 & 5803 & 5984 & 6116 & 5891 & 6506 & 6133 & 6291 & 5582 & 6382 & & $21.36^{* *}$ \\
\hline
\end{tabular}

HD: Heading time; SS: stalks per square meter; ES: ear per square meter; MT: maturation time; PH: plant height; SL: spike length; NSS: number of spikelets per spike; NGS: number of grains per spike; SY: spike yield; SPAD: leaf chlorophyll content; VIT: vitreous kernels; HW: hectoliter weight; TGW: 1000-grain weight; PC: protein content; SC: semolina color; SDS: mini sedimentation; WG: wet gluten; YLD: yield.

$*$,**Significant at the 0.05 and 0.01 probability level, respectively.

The number of HD days was calculated from seed germination to heading period (50\%) and MT was calculated from seed germination to plant maturity $(100 \%)$.

of 10 durum wheat cultivars are displayed in Table 3, and the pairwise correlations among traits of the 10 durum wheat cultivars are shown in Table 4. These data were used to create a GT biplot (Figure 1), and the goodness of fit of the biplot is relatively strong because it represents $61.50 \%$ of the variation.

The relationships among traits in Figure 1A are visualized by genotype profiles. A biplot illustrated as a graph can be bi-directionally interpreted in different ways (Yan and Rajcan, 2002; Yan and Tinker, 2006; Dogan et al., 2016). Firstly, the cosine of the angle between the vectors of the two properties approaches the Pearson correlation between them. Therefore, an angle $<90^{\circ}$ shows a positive correlation, an angle $>90^{\circ}$ shows a negative correlation, and an angle of $90^{\circ}$ shows a zero correlation. Secondly, the length of the vector is an approximation of trait variation. Thirdly, the

Table 4. Pairwise correlations among traits of 10 durum wheat cultivars.

\begin{tabular}{|c|c|c|c|c|c|c|c|c|c|c|c|c|c|c|c|c|c|}
\hline & YLD & HD & SS & ES & MT & PH & SL & NSS & NGS & SY & VIT & SPAD & TGW & HW & PC & $\mathrm{SC}$ & SDS \\
\hline HD & -0.44 & & & & & & & & & & & & & & & & \\
\hline SS & 0.78 & 0.02 & & & & & & & & & & & & & & & \\
\hline ES & 0.76 & 0.01 & 0.93 & & & & & & & & & & & & & & \\
\hline MT & 0.04 & 0.40 & 0.19 & 0.08 & & & & & & & & & & & & & \\
\hline PH & 0.73 & -0.33 & 0.59 & 0.53 & 0.12 & & & & & & & & & & & & \\
\hline SL & 0.11 & -0.11 & 0.07 & 0.15 & -0.17 & -0.05 & . & & & & & & & & & & \\
\hline NSS & 0.02 & 0.07 & -0.08 & -0.07 & 0.20 & -0.01 & 0.41 & . & & & & & & & & & \\
\hline NGS & 0.13 & -0.09 & -0.06 & -0.09 & 0.07 & 0.30 & -0.08 & 0.12 & . & & & & & & & & \\
\hline SY & 0.37 & -0.43 & 0.03 & 0.04 & -0.16 & 0.38 & 0.20 & 0.11 & 0.65 & . & & & & & & & \\
\hline VIT & 0.03 & 0.33 & 0.29 & 0.35 & -0.21 & -0.10 & 0.23 & -0.11 & -0.37 & -0.24 & . & & & & & & \\
\hline SPAD & 0.39 & 0.06 & 0.51 & 0.64 & -0.32 & 0.20 & 0.26 & -0.09 & -0.10 & 0.10 & 0.45 & . & & & & & \\
\hline TGW & 0.21 & -0.48 & -0.12 & -0.09 & -0.03 & 0.18 & 0.25 & 0.18 & 0.19 & 0.57 & -0.34 & -0.11 & . & & & & \\
\hline HW & 0.21 & -0.16 & 0.09 & 0.14 & 0.11 & 0.12 & 0.02 & 0.19 & 0.05 & 0.11 & 0.00 & 0.09 & 0.12 & . & & & \\
\hline PC & 0.08 & 0.28 & 0.40 & 0.45 & -0.21 & 0.01 & 0.17 & -0.27 & -0.33 & -0.28 & 0.56 & 0.59 & -0.35 & -0.28 & . & & \\
\hline $\mathrm{SC}$ & -0.03 & 0.05 & 0.15 & 0.13 & -0.12 & -0.10 & 0.10 & -0.15 & -0.25 & -0.29 & 0.29 & -0.03 & -0.29 & -0.29 & 0.37 & . & \\
\hline SDS & -0.29 & 0.26 & -0.09 & -0.12 & -0.09 & -0.22 & 0.05 & -0.24 & -0.25 & -0.35 & 0.38 & -0.01 & -0.31 & -0.34 & 0.41 & 0.53 & . \\
\hline WG & 0.10 & 0.30 & 0.38 & 0.38 & 0.07 & 0.07 & 0.07 & -0.24 & -0.28 & -0.29 & 0.45 & 0.44 & -0.37 & -0.22 & 0.87 & 0.27 & 0.38 \\
\hline
\end{tabular}

YLD: Yield; HD: heading time; SS: stalks per square meter; ES: ears per square meter; MT: maturation time; PH: plant height; SL: spike length; NSS: number of spikelets per spike; NGS: number of grains per spike; SY: spike yield; VIT: vitreous kernels; SPAD: leaf chlorophyll content; TGW: 1000-grain weight; HW: hectoliter weight; PC: protein content; SC: semolina color; SDS: mini sedimentation; WG: wet gluten.

The correlation thresholds are 0.93 for $\mathrm{P}<0.5$ and 0.10 for $\mathrm{P}<0.1$. 
Figure 1. (A) Relationship of genotype $\times$ trait (GT) based on two seasons of data, (B) stability of GT based in two seasons of data, (C) which-won-where/what of GT biplot based on data across seasons, and (D) comparison of GT based in 2 years of data.
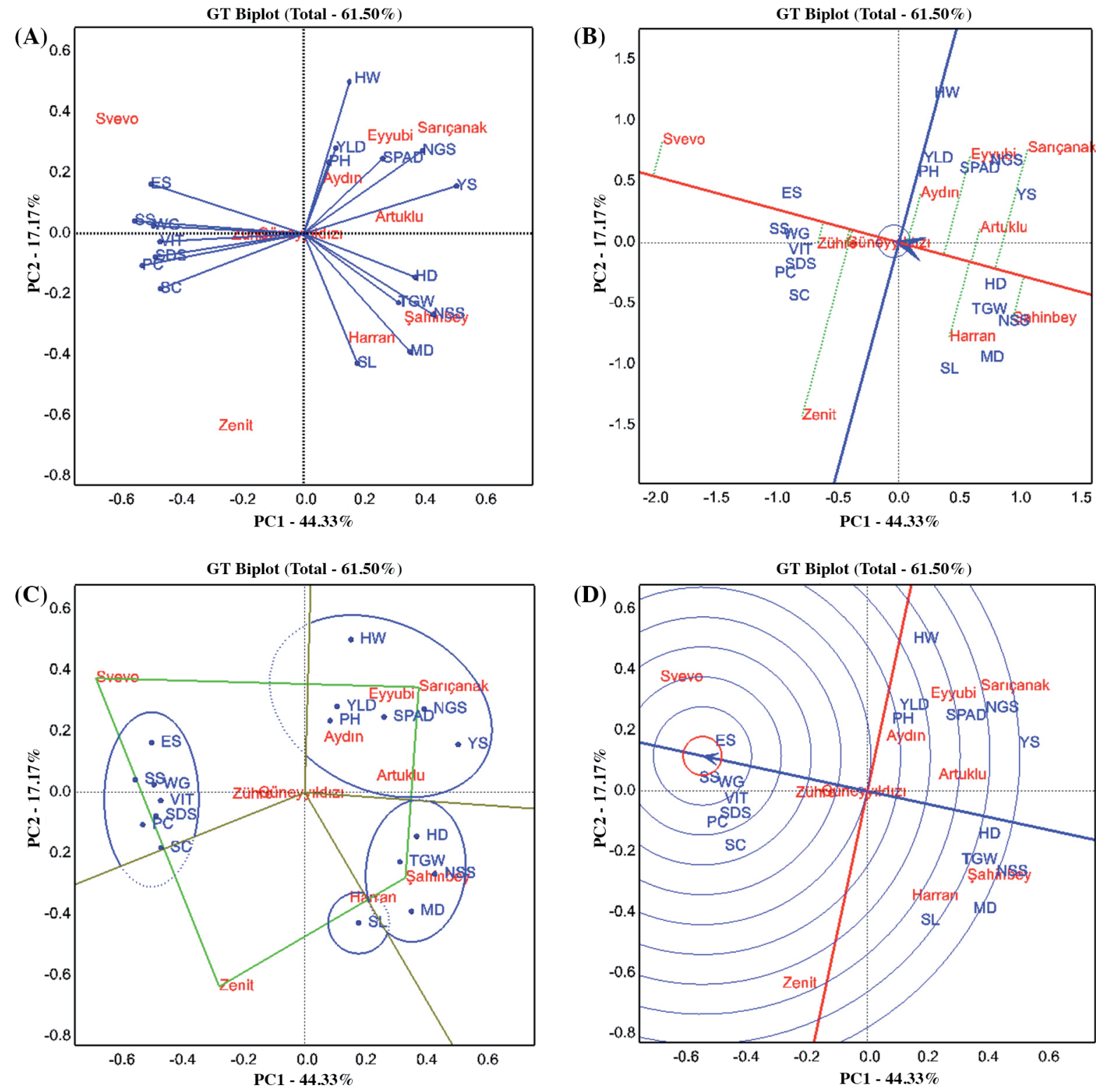

YLD: Yield; HD: heading time; SS: stalks per square meter; ES: ears per square meter; MT: maturation time; PH: plant height; SL: spike length; NSS: number of spikelets per spike; NGS: number of grains per spike; SY: yield of spike; SPAD: leaf chlorophyll content; VIT: vitreous kernels; HW: hectoliter weight; TGW: 1000-grain weight; PC: protein content; SC: semolina color; SDS: mini sedimentation; WG: wet gluten.

angle between the vector of any genotype and any trait gives information about the state of the genotypes. If the angle is quite acute or if the angle is too large, this indicates that the genotype is below the mean for that trait. Finally, the vector length of a genotype indicates its strength or weakness for all trait profiles. According to these principles described in the GT biplot technique, the following observations can be made about Figure 1A. Grain yield was positively and highly correlated with PH, SPAD, HW, NGS, and SY, but it was negatively correlated with quality traits (ES, SS, WG, VIT, SDS, PC, and SC); however, it was not associated with physiological traits (HD, TGW, NSS, and MT). On the other hand, the explanations are confirmed by correlation values in Tables 3 and 4 .

Figure 1B shows a vertical mean axis and a horizontal stability axis based on trait values; the genotypes are evaluated based on these two axes. If the genotypes are located below the vertical axis, they are undesirable. If they are located 
above the vertical axis, they are desirable genotypes. On the other hand, genotypes located near or at the center of the horizontal line are stable and unstable if they move away from the horizontal line (Kendal and Sayar, 2016; Oral et al., 2018). According to Figure 1B, 'Güneyyildizi' and 'Zühre' are quite stable because they are located at the center of the horizontal axis. 'Svevo' is very favorable because it is located near the center of the horizontal axis and on all traits. 'Zenit' is the most unstable because this variety is located far from the center of the horizontal axis. 'Şahinbey', 'Sariçanak', 'Harran', and 'Eyyubi' are undesirable genotypes because they are located under the vertical axis line. Other genotypes (e.g., 'Zenit')that are located above the axis vertical line are desirable based on trait profiles.

The polygon of the which-won-where/what of the GT biplot based on data across seasons is shown in Figure 1C. By dividing the figure with thick axes from the center and separating each zone with two thick lines, sectors are obtained and indicated by correlative numbers $(1,2,3$, etc). If the genotypes and properties are located in the same sector when starting from the lower right part of the graph, they are closely related (Yan and Tinker, 2006; Kendal and Dogan, 2015). According to this description, Figure 1C is divided into four sectors, and different traits are associated with different cultivars in each sector. 'Şahinbey' won in sector 1 and was correlated with HD, TGW, NSS, and MT. 'Sariçanak' won in sector 2 where 'Artuklu', 'Eyyubi', and 'Aydin' were also located and was correlated with PH, SPAD, HW, NGS, and SY. 'Svevo' won in sector 3, which is the same sector where 'Zühre' and 'Güneyyildizi' were located, and it was correlated with ES, SS, WG, VIT, SDS, PC, and SC. 'Zenit' won in sector 4 and was correlated with SL.

The discrimination and representativeness of genotypes based on traits are displayed in Figure 1D; it shows a representative "ideal center" over the property mean values and allows evaluating genotypes according to their nearness or distance to this center (Yan et al., 2000; Yan and Tinker, 2005; Kendal et al., 2016). The most ideal genotypes are located in the center, whereas genotypes located on the mean vertical axis, but far from the center, are ideal; genotypes located below the vertical axis are undesirable. According to Figure 1C, 'Zühre' and 'Svevo' are more ideal than 'Zenit' because they are the nearest varieties to the "ideal center", while 'Şahinbey' and 'Sariçanak' are located under the vertical axis and far from "ideal center" and are undesirable varieties.

\section{The genotype $\times$ yield $\times$ trait biplot combination}

The GYT biplot, as a combination of yield with any other trait, is used to measure how grain yield is combined with that trait in cultivars. Therefore, the GYT biplot technique was used to determine when the value of a trait of any genotype was low and grain yield was high or vice versa and whether the results were affected by the combination or if there was any change in their ranking. As a result, when trait and yield values entered the combination, data changed as did cultivar ranking. Therefore, a greater value is always desirable in the GYT table. The values of 10 durum wheat cultivars across 2 yr in four locations are listed in Table 5. Values in the GYT table (Table 5) were generated from the GT table (Table 3). In the GYT table, data in each column consist of a yield-trait combination. The standardized GYT values and SI of 10 durum wheat cultivars across $2 \mathrm{yr}$ in four locations are shown in Table 6 . The genotypes were quite compatible with the biplot and represented $76.09 \%$ of the total variation (PC1 50.01\%, PC2 26.08\%). Each figure was described in the GT biplot section. These descriptions also described GYT biplot patterns. For this reason, the GYT biplot will not be described again, but only the results obtained from the GYT biplot shapes are given below.

Based on the principles described in the GYT biplot technique, the following observations can be made about the relationships between the yield-trait combinations illustrated in Figure 2A. According to this figure, all yield-trait combinations tend to correlate positively with each other because they have the yield component, shown by the acute angle between the vectors. This is an important feature of the GYT biplot technique (Figure 2) compared with the GT biplot (Figure 1). The yield-trait combinations graphically provide the opportunity for genotypes to be ranked in a more meaningful way. However, the relationships between the two traits in the GT biplot technique (Figure 1A) are not the same as for GYT; for example, there is a positive correlation between YLD and PH and a negative correlation between YLD and PC and SC (Figure 1A and Table 3). The same relationship can also be seen with the GYT biplot technique, as indicated by lower correlation values and acute angles between YLD $\times \mathrm{PH}, \mathrm{YLD} \times \mathrm{PC}$, and YLD $\times \mathrm{SC}$. On the other hand, 'Svevo' was correlated with the YLD $\times$ PH and YLD $\times$ SDS combinations, 'Zühre' with the YLD $\times$ VIT, YLD $\times$ $\mathrm{PC}, \mathrm{YLD} \times \mathrm{TGW}, \mathrm{YLD} \times \mathrm{ES}, \mathrm{YLD} \times \mathrm{WG}$, and YLD $\times$ SS combinations, and 'Sariçanak' was correlated with the YLD $\times$ $\mathrm{SL}, \mathrm{YLD} \times \mathrm{PH}, \mathrm{YLD} \times \mathrm{SPAD}, \mathrm{YLD} \times \mathrm{HW}, \mathrm{YLD} \times \mathrm{NGS}, \mathrm{YLD} \times \mathrm{SY}, \mathrm{YLD} \times \mathrm{HD}, \mathrm{YLD} \times \mathrm{TGW}, \mathrm{YLD} \times \mathrm{NSS}$, and YLD $\times$ MT combinations. Therefore, these three cultivars have good results based on GY trait combinations. 
Table 5. Standardized genotype $\times$ yield $\times$ trait $($ GYT) data and superiority index (SI) for 10 durum wheat cultivars across 2 years in four environments.

\begin{tabular}{|c|c|c|c|c|c|c|c|c|c|c|c|}
\hline \multirow{2}{*}{$\begin{array}{l}\text { Yield } \times \text { Trait } \\
\text { Cross }\end{array}$} & \multicolumn{10}{|c|}{ Cultivars } & \multirow[b]{2}{*}{ SD } \\
\hline & $\mathrm{C} 1$ & $\mathrm{C} 2$ & C3 & $\mathrm{C} 4$ & C5 & C6 & C7 & $\mathrm{C} 8$ & C9 & $\mathrm{C} 10$ & \\
\hline $\mathrm{YLD} \times \mathrm{HD}$ & 0.98 & 0.97 & 0.99 & 1.02 & 0.98 & 1.07 & 0.99 & 1.04 & 0.92 & 1.05 & 1.00 \\
\hline YLD × SS & 0.94 & 0.95 & 0.95 & 1.04 & 0.96 & 1.06 & 1.10 & 0.99 & 0.95 & 1.06 & 1.00 \\
\hline $\mathrm{YLD} \times \mathrm{ES}$ & 0.92 & 0.96 & 0.99 & 1.01 & 0.98 & 1.06 & 1.11 & 0.95 & 0.93 & 1.09 & 1.00 \\
\hline YLD × MT & 0.98 & 0.96 & 0.98 & 1.01 & 0.98 & 1.07 & 1.00 & 1.04 & 0.92 & 1.06 & 1.00 \\
\hline YLD × PH & 1.05 & 1.06 & 1.01 & 1.03 & 0.93 & 1.00 & 1.00 & 1.02 & 0.85 & 1.04 & 1.00 \\
\hline $\mathrm{YLD} \times \mathrm{SL}$ & 1.03 & 0.89 & 1.00 & 1.01 & 0.98 & 1.05 & 0.90 & 1.06 & 1.02 & 1.06 & 1.00 \\
\hline $\mathrm{YLD} \times \mathrm{NSS}$ & 0.98 & 0.99 & 0.99 & 0.96 & 1.00 & 1.12 & 0.90 & 1.06 & 0.96 & 1.05 & 1.00 \\
\hline YLD $\times$ NGS & 1.02 & 0.99 & 1.02 & 1.03 & 0.98 & 1.19 & 0.92 & 0.96 & 0.85 & 1.04 & 1.00 \\
\hline $\mathrm{YLD} \times \mathrm{SY}$ & 1.04 & 0.94 & 1.03 & 1.02 & 0.99 & 1.13 & 0.95 & 1.07 & 0.85 & 0.97 & 1.00 \\
\hline $\mathrm{YLD} \times \mathrm{SPAD}$ & 0.99 & 0.96 & 1.06 & 0.96 & 0.97 & 1.08 & 1.00 & 1.03 & 0.90 & 1.05 & 1.00 \\
\hline YLD × VIT & 0.98 & 0.94 & 0.97 & 1.03 & 0.91 & 1.01 & 1.07 & 1.03 & 0.95 & 1.11 & 1.00 \\
\hline YLD × HW & 0.98 & 0.97 & 1.00 & 1.01 & 0.95 & 1.09 & 1.01 & 1.03 & 0.91 & 1.05 & 1.00 \\
\hline YLD $\times$ TGW & 0.97 & 0.91 & 1.01 & 1.00 & 1.02 & 1.05 & 0.97 & 1.18 & 0.90 & 0.98 & 1.00 \\
\hline YLD $\times$ PC & 0.96 & 0.98 & 0.96 & 1.03 & 0.97 & 1.02 & 1.06 & 1.01 & 0.94 & 1.06 & 1.00 \\
\hline $\mathrm{YLD} \times \mathrm{SC}$ & 0.92 & 0.94 & 0.91 & 1.05 & 0.98 & 1.06 & 1.09 & 0.93 & 1.05 & 1.07 & 1.00 \\
\hline $\mathrm{YLD} \times \mathrm{SDS}$ & 0.86 & 0.78 & 1.12 & 1.12 & 0.96 & 0.72 & 1.27 & 0.74 & 1.14 & 1.30 & 1.00 \\
\hline $\mathrm{YLD} \times \mathrm{WG}$ & 0.98 & 0.99 & 0.96 & 1.02 & 0.95 & 1.00 & 1.07 & 1.00 & 0.93 & 1.09 & 1.00 \\
\hline Mean (SI) & 0.97 & 0.95 & 1.00 & 1.02 & 0.97 & 1.05 & 1.02 & 1.01 & 0.94 & 1.07 & \\
\hline
\end{tabular}

YLD: Yield; HD: heading time; SS: stalks per square meter; ES: ears per square meter; MT: maturation time; PH: plant height; SL: spike length; NSS: number of spikelets per spike; NGS: number of grains per spike; SY: spike yield; VIT: vitreous kernels; SPAD: leaf chlorophyll content; TGW: 1000-grain weight; HW: hectoliter weight; PC: protein content; SC: semolina color; SDS: mini sedimentation; WG: wet gluten; SD: standard deviation.

Table 6. Genotype $\times$ yield $\times$ trait $($ GYT) data for 10 durum wheat cultivars across 2 years in four environments.

\begin{tabular}{|c|c|c|c|c|c|c|c|c|c|c|c|}
\hline Yield $\times$ Trait & $\mathrm{C} 1$ & $\mathrm{C} 2$ & $\mathrm{C} 3$ & $\mathrm{C} 4$ & C5 & C6 & C7 & C8 & C9 & $\mathrm{C} 10$ & Mean \\
\hline YLD $\times H D$ & 735701 & 730988 & 743728 & 764469 & 737263 & 809041 & 744777 & 780297 & 692638 & 792319 & 753122 \\
\hline $\mathrm{YLD} \times \mathrm{SS}$ & 3375809 & 3399248 & 3425039 & 3748764 & 3458050 & 3808330 & 3942376 & 3570219 & 3408248 & 3809036 & 3594512 \\
\hline $\mathrm{YLD} \times \mathrm{ES}$ & 2709507 & 2824395 & 2917314 & 2986206 & 2887384 & 3105832 & 3252412 & 2784810 & 2747180 & 3213949 & 2942899 \\
\hline $\mathrm{YLD} \times \mathrm{MT}$ & 976751 & 961110 & 981788 & 1008334 & 978600 & 1073571 & 996998 & 1043935 & 923750 & 1055960 & 1000080 \\
\hline $\mathrm{YLD} \times \mathrm{PH}$ & 606132 & 613841 & 584211 & 594183 & 537714 & 577858 & 575161 & 586 & 491876 & & 576510 \\
\hline $\mathrm{YLD} \times \mathrm{SL}$ & 42115 & 36303 & 40872 & 4111 & 39832 & 42719 & & 43204 & 41 & 43236 & 40780 \\
\hline YLD $\times$ NSS & 117572 & 119141 & 119198 & 1161 & 120871 & 134806 & 108439 & 1273 & 115 & 126117 & 120538 \\
\hline$Y L D \times N G S$ & 304118 & 295405 & 304915 & 30712 & 293377 & 355661 & 273 & 287957 & 253334 & 309728 & 298541 \\
\hline YLD × SY & 13560 & 12278 & 13500 & 133 & 12965 & 14825 & 12423 & 14031 & 11134 & 12723 & 13082 \\
\hline YLD $x$ & 285283 & 276510 & 305551 & 27692 & 278835 & 310949 & 287197 & 295320 & 260101 & 301821 & 287850 \\
\hline YLD × VIT & 552985 & 534535 & 551783 & 584597 & 513497 & 570180 & 604983 & 585723 & 539847 & 628871 & 566700 \\
\hline YLD $\times$ HW & 497198 & 493177 & 508005 & 512156 & 483483 & 556132 & 513783 & 521040 & 461239 & 533774 & 507999 \\
\hline YLD $\times$ TGW & 262980 & 245636 & 273872 & 270507 & 276515 & 282402 & 263355 & 317423 & 244403 & 264289 & 270138 \\
\hline YLD × PC & 81147 & 82665 & 80984 & 87111 & 82388 & 86658 & 89475 & 85576 & 79843 & 89792 & 84564 \\
\hline $\mathrm{YLD} \times \mathrm{SC}$ & 119678 & 121273 & 118317 & 1359 & 126907 & 137225 & 141644 & 120060 & 135944 & 139211 & 129618 \\
\hline & 32900 & & & & & & & & & 49807 & 38403 \\
\hline $\mathrm{YLD} \times \mathrm{WG}$ & 162164 & 163340 & 159106 & 169234 & 157509 & 164777 & 176528 & 164756 & 153842 & 180627 & 165188 \\
\hline
\end{tabular}

YLD: Yield; HD: heading time; SS: stalks per square meter; ES: ears per square meter; MT: maturation time; PH: plant height; SL: spike length; NSS: number of spikelets per spike; NGS: number of grains per spike; SY: spike yield; VIT: vitreous kernels; SPAD: leaf chlorophyll content; TGW: 1000-grain weight; HW: hectoliter weight; PC: protein content; SC: semolina color; SDS: mini sedimentation; WG: wet gluten.

The stability and superiority effects of cultivars by GYT are found in Figure 2B. The horizontal line with an arrow indicates the stability line of the combination and evaluates the cultivars based on this line. On the other hand, genotype superiority is determined by the vertical line with no arrow. Thus, the stability and superiority analysis indicated that 'Güneyyildizi' was the most stable, while 'Zühre' and 'Sariçanak' were only superior cultivars. Moreover, 'Zenit', 'Artuklu', and 'Eyyubi' were unstable and undesirable genotypes because they were located under the mean line of multiple traits. The SI ranks cultivars with the mean of all traits (Figure 2B, Table 6); high values of SI (1.09) indicate the best cultivar ('Zühre') and low values of SI (0.93) indicate a weak cultivar ('Zenit'). 
Figure 2. (A) Relationship of genotype $\times$ yield $\times$ trait (GYT) biplot based on combining two seasons of data, (B) stability of GYT based on combining two seasons of data, (C) which-won-where/what of GYT based on data across seasons, and (D) comparison of GYT based on combining two seasons of data.
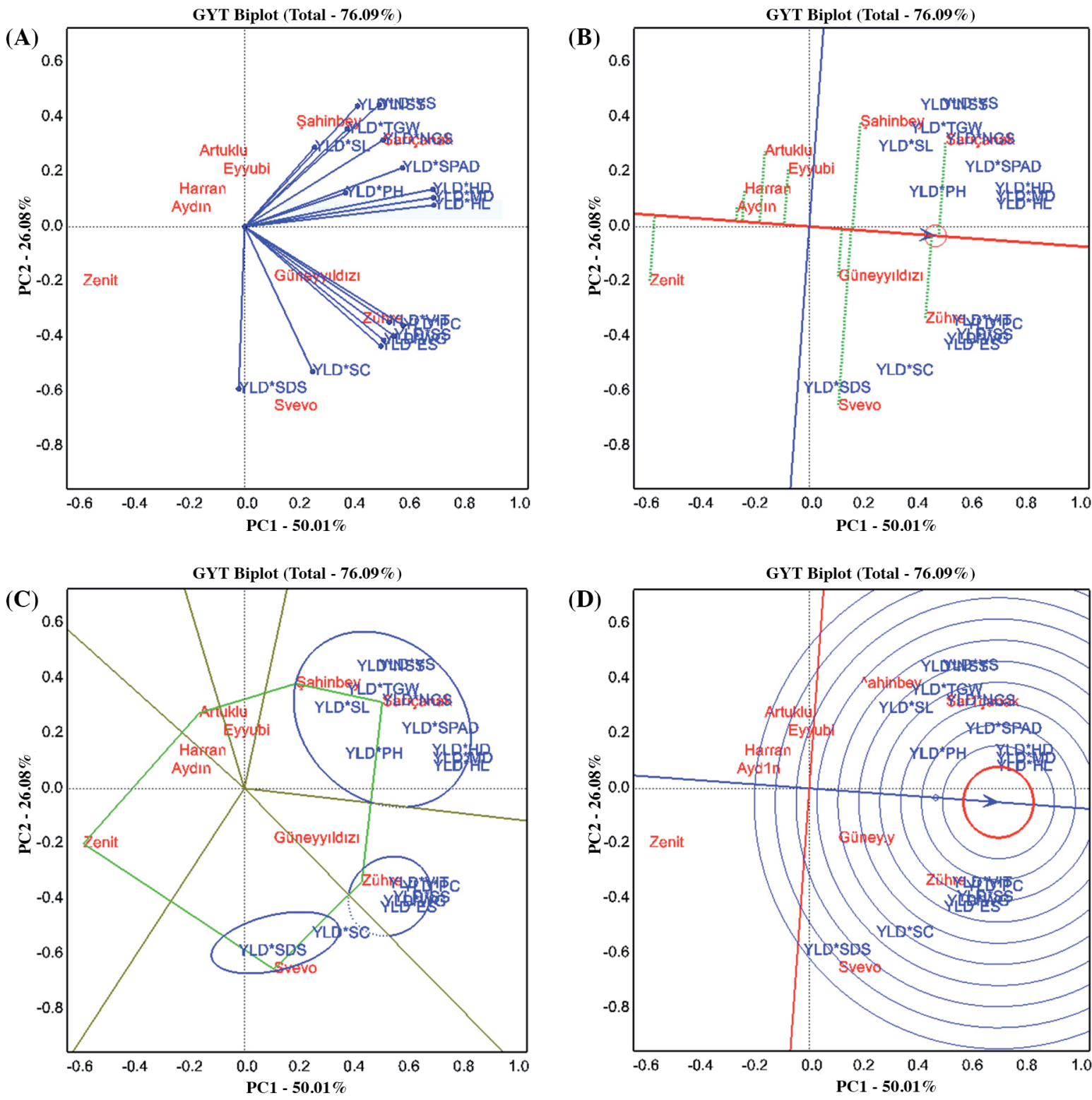

YLD: Yield; HD: heading time; SS: stalks per square meter; ES: ears per square meter; MT: maturation time; PH: plant height; SL: spike length; NSS: number of spikelets per spike; NGS: number of grains per spike; SY: yield of spike; VIT: vitreous kernels; SPAD: leaf chlorophyll content; TGW: 1000-grain weight; HW: hectoliter weight; PC: protein content; SC: semolina color; SDS: mini sedimentation; WG: wet gluten.

Exhibited cultivar trait profiles by which-won-where sector analysis in the GYT biplot are illustrated in Figure 2C. The most effective cultivar associated with trait profiles in each sector is indicated by a polygon peak. In sector analysis, the figure was divided into six main sectors and the combinations were located in their sector as a group. 'Güneyyildizi' and 'Svevo' are located in the same sector (Sector 1) with YLD $\times$ PH and YLD $\times$ SDS combinations, whereas 'Svevo' is located in the vertex (top) of this sector and is the winning cultivar for the combination located in same sector. 'Zühre' is only located in sector 2 with the YLD $\times$ VIT, YLD $\times$ PC, YLD $\times$ TGW, YLD $\times E S, Y L D \times W G$, and YLD $\times$ SS combinations and wins the vertex of this sector. 'Sariçanak' is located at the vertex of the polygon in sector 3 and is correlated with the YLD $\times$ SL, YLD $\times$ PH, YLD $\times$ SPAD, YLD $\times$ HW, YLD $\times$ NGS, YLD $\times$ SY, YLD $\times$ HD, YLD $\times$ 
TGW, YLD $\times$ NSS, and YLD $\times$ MT combinations; 'Şahinbey' is also located in this sector with 'Sariçanak'. The other cultivars (Artuklu, Eyyubi, Harran, Aydin, and Zenit) are located in other sectors (4, 5, and 6) and are not correlated with any yield-trait combination. On the other hand, there are three groups, in Figure $2 \mathrm{C}$ presented as three circles between combinations.

Discrimination and representativeness of genotypes based on the GYT combination is indicated in Figure 2D and provides a representative "ideal center" over the GYT mean values. Figure $2 \mathrm{C}$ predicts that 'Sariçanak' and 'Zühre' are the ideal cultivars because they are located nearest to the "ideal center"; 'Şahinbey', 'Güneyyildizi', and 'Svevo' are desirable for the GYT combination, while 'Artuklu', 'Eyyubi', 'Harran', 'Aydin', and 'Zenit' are undesirable genotypes because they are located under the mean combination values of the vertical line.

\section{DISCUSSION}

The main purpose of multi-environment experiments in durum wheat is to identify superior varieties based on multiple traits and mega environments. Given the unpredictable environmental factors in the GE interaction studies, different models (GE, GE interaction, and AMMI), were developed to elucidate the effect of genotype, environment, or interaction; they are still used in breeding studies (Kendal, 2015; Kendal and Sayar, 2016). In addition, the GT biplot technique has been used for a long time by many researchers to understand the effect of genotype and environment on the relationships between agronomic, physiological and quality characters, and yield (Yan and Tinker, 2006; Kendal and Dogan, 2015; Akcura et al., 2016; Oral et al., 2018). The GT biplot is used to compare varieties based on multiple traits and to define them based on these traits. This technique does not suffice to determine the effect of combining all the traits on yield under multiple environmental conditions, while the relationship between each trait and yield can be determined. Therefore, the GYT biplot technique has been developed to determine the effect of combining all traits with yield under multiple environmental conditions. However, publications based on multiple traits combined with grain yield (GYT) in different environments to evaluate the varieties are limited (Yan and Frégeau-Reid, 2018).

In durum wheat, cultivars are only preferred by farmers when they have high grain yield: however, high yield has been affected by combining many traits. Thus, the GYT biplot provides breeders the opportunity to evaluate cultivars and identify both superior cultivars and the superiority index. The GYT methodology reveals all the factors that affect high efficiency by determining the effect of each trait that affects high efficiency under multiple environmental conditions; it is therefore used by breeders. If the varieties are registered with a selection based on combining traits, obtained from multiple locations, with yield, they will then be quite stable in terms of all traits and yield for similar environments. For this purpose, the GYT biplot methodology has been recently developed and used by a few researchers to evaluate the values obtained from combining multiple traits with yield and multiple environments in breeding studies. The GYT biplot approach has been reported as a comprehensive and effective method; it classifies genotypes according to their levels in the combination with target characteristics and graphically ranks genotypes for their strengths, weaknesses, and in different plants (Yan and Frégeau-Reid, 2018).

In durum wheat cultivation, some traits (ES, NSS, and SY) directly increase yield and some traits indirectly increase yield (SS, HD, MT, SPAD, NGS, and SL), while some quality parameters affect seed quality (TGW, HW, VIT, PC, SC, SDS, and WG). Yield is not the only trait in durum wheat studies that can determine the effectiveness of a single genotype; quality traits and the length of time between HD and MT are valuable for breeders when combined with high yield levels. For example, a durum wheat cultivar is not valuable for breeders if its quality is high but its resistance to drought and yield are low. However, if cultivar yield is high, the cultivar becomes valuable if it has good agronomic and quality traits. Therefore, in selecting the best cultivars, the yield-trait combination effects are more meaningful than the effects of individual traits. In the GT biplot technique, a high value (Table 3, Figure 1B) makes the average tester coordinate (ATC) appear nonsignificant in some cases, while in the GYT biplot technique, it makes the ATC appear as a meaningful and effective tool because it ranks cultivars based on various yield-trait combinations and indicates their strengths and weaknesses (Figure 2B, Table 5).

The GT biplot technique was used to construct Figure 1 (A-D) using the data in Table 3, while the GYT biplot technique was used in Figure 2 (A-D) using the data in Table 5. The bulgur and macaroni industries demand durum wheat products that have the best yellow color and good processing properties, while farmers demand highly productive and high quality 
varieties. As in other durum wheat products, it is very difficult to identify both high yield and high quality varieties, and this situation becomes more complicated when different environmental problems are combined. These complex problems can only be solved by using the GYT biplot methodology. The examination of genotypes depended on the SI and GYT combination); the GYT results (Figure 2) show that poor and strong cultivars can be determined by GYT and that this technique is better than the GT biplot (Figure 1). On the other hand, in the GTY biplot, 'Güneyyildizi' is stable for all traits and 'Zühre' for quality traits, while 'Sariçanak' for agronomic traits. Therefore, the present study found that the GYT biplot technique is an appropriate method to determine the most suitable genotypes for all traits in durum wheat cultivars.

\section{CONCLUSIONS}

The objectives of the present study suggest that there are more reasons to use genotype $\times$ yield $\times$ trait $(\mathrm{GYT})$ combinations in multi-location, multi-year, and multi-trait studies of durum wheat. Therefore, if GYT combinations are significant, they should be investigated. In the GYT technique, there is a strong and narrow (usually positive) relationship between combinations, whereas the GT biplot technique determines that there is a weak and wide (both positive and negative) relationship between grain yield and traits. The GYT biplot technique provides information on the general adaptability of cultivars. 'Güneyyildizi' was stable for all traits and 'Zühre' for quality traits, while 'Sariçanak' was stable for agronomic traits. Cultivar stability and the best cultivar are clearly observed with the GYT biplot technique.

\section{REFERENCES}

Akcura, M., Kokten, K., Akcacik, A.G., and Aydogan S. 2016. Pattern analysis of Turkish bread wheat landraces and cultivars for grain and flour quality. Turkish Journal of Field Crops 21(1):120-130.

Baxevanos, D., Korpetis, E., Irakli, M., and Tsialtas, I.T. 2017. Evaluation of a durum wheat selection scheme under Mediterranean conditions: adjusting trial locations and replications. Euphytica 213(4):82.

Dogan, Y., Kendal, E., and Oral, E. 2016. Identifying of relationship between traits and grain yield in spring barley by GGE Biplot analysis. Agriculture \& Forestry/Poljoprivreda i Sumarstvo 62(4):239-252.

Giunta, F., De Vita, P., Mastrangelo, A.M., Sanna, G., and Motzo R. 2018. Environmental and genetic variation for yield-related traits of durum wheat as affected by development. Frontiers in Plant Science 9:8.

Karaman, M. 2019. Evaluation of bread wheat genotypes in irrigated and rainfed conditions using biplot analysis. Applied Ecology and Environmental Research 17(1):1431-1450.

Kendal, E. 2015. Relationship between chlorophyll and other features in durum wheat (Triticum turgidum L. var. durum) using SPAD and biplot analyses. Journal of Agricultural Science and Technology 17:1873-1886.

Kendal, E., and Dogan, Y. 2015. Stability of a candidate and cultivars (Hordeum vulgare L.) by GGE biplot analysis of multienvironment yield trials in spring barley. Agriculture \& Forestry 61(4):307-318.

Kendal, E., and Sayar, M.S. 2016. The stability of some spring triticale genotypes using biplot analysis. The Journal of Animal \& Plant Sciences 26(3):754-765.

Kendal, E., Tekdal, S., and Sayar, M.S. 2016. Assessment of the impact of ecological factors on yield and quality parameters in triticale using GGE biplot and AMMI analysis. Pakistan Journal of Botany 48(5):1903-1913.

Kilic, H. 2014. Additive main effect and multiplicative interactions (AMMI) Analysis of grain yield in barley genotypes across environments. Journal of Agricultural Sciences 20:337-344.

Kilic, H., Sanal, T., Erdemci, I., and Karaca, K. 2017. Screening bread wheat genotypes for high molecular weight glutenin subunits and some quality parameters. Journal of Agricultural Science and Technology 19(6):1393-1404.

Magallanes-López, A.M., Ammar, K., Morales-Dorantes, A., González-Santoyo, H., Crossa, J., and Guzmán, C. 2017. Grain quality traits of commercial durum wheat varieties and their relationships with drought stress and glutenins composition. Journal of Cereal Science 75:1-9.

Mohammadi, R., Armion, M., Zadhasan, E., Ahmadi, M.M., and Amri, A. 2018. The use of AMMI model for interpreting genotypexenvironment interaction in durum wheat. Experimental Agriculture 54(5):670-683.

Mohammadi, R., Farshadfar, E., and Amri, A. 2015. Interpreting genotype $\times$ environment interactions for grain yield of rainfed durum wheat in Iran. The Crop Journal 3(6):526-535.

Oral, E., Kendal, E., and Dogan, Y. 2018. Selection the best barley genotypes to multi and special environments by AMMI and GGE biplot models. Fresenius Environmental Bulletin 27(7):5179-5187.

Pinheiro, N., Costa, R., Almeida, A.S., Coutinho, J., Gomes, C., and Macas, B. 2013. Durum wheat breeding in Mediterranean environments-influence of climatic variables on quality traits. Emirates Journal of Food and Agriculture 25(12):962-974. 
Sadeghzadeh, B., Mohammadi, R., Ahmadi, H., Abediasl, G.R., Ahmadi, M.M., Mohammadfam, M., et al. 2018. GGE biplot and AMMI application in the study of adaptability and grain yield stability of durum lines under dryland conditions. Environmental Stresses in Crop Sciences 11(2):241-260.

Sissons, M., Ovenden, B., Adorada, D., and Milgate, A. 2014. Durum wheat quality in high-input irrigation systems in southeastern Australia. Crop and Pasture Science 65(5):411-422.

Tekdal, S., and Kendal, E. 2018. AMMI model to assess durum wheat genotypes in multi-environment trials. Journal of Agricultural Science and Technology 20:153-166.

Yan, W., and Frégeau-Reid, J. 2018. Genotype by Yield×Trait (GYT) Biplot: a novel approach for genotype selection based on multiple traits. Scientific Reports 8:8242.

Yan, W., Hunt, L.A., Sheng, Q., and Szlavnics, Z. 2000. Cultivar evaluation and mega-environment investigation based on the GGE biplot. Crop Science 40(3):597-605.

Yan, W., and Rajcan, I.R. 2002. Biplot analysis of test sites and trait relations of soybean in Ontario. Canadian Journal of Plant Science 42:11-20

Yan, W., and Tinker, N.A. 2005. An integrated biplot analysis system for displaying, interpreting, and exploring genotypex environment interaction. Crop Science 45(3):1004-1016.

Yan, W., and Tinker, N.A. 2006. Biplot analysis of multi-environment trial data: Principles and applications. Canadian Journal of Plant Science 86(3):623-645. 